(C)2008 IEEE. Personal use of this material is permitted. However, permission to reprint/republish this material for advertising or promotional purposes or for creating new collective works for resale or redistribution to servers or lists, or to reuse any copyrighted component of this work in other works must be obtained from the IEEE. 


\section{Blind Spatial Signature Estimation Using Time-Varying User Power Loading and Parallel Factor Analysis}

\author{
Yue Rong Sergiy A. Vorobyov \\ Department of Communication Systems \\ University of Duisburg-Essen \\ Duisburg, Germany
}

\author{
Alex B. Gershman \\ Dept. of ECE \\ McMaster University \\ Hamilton, Ontario, Canada
}

\author{
Nicholas D. Sidiropoulos \\ Dept. of ECE \\ Technical University of Crete \\ Chania, Crete, Greece
}

\begin{abstract}
The problem of blind user spatial signature estimation using the parallel factor (PARAFAC) analysis model is addressed in the context of applications to wireless communications. A time-varying user power loading in the uplink mode is proposed to enable identifiability and application of PARAFAC analysis. Identifiability issues are carefully studied and two blind spatial signature estimation algorithms are developed. The first technique is based on the PARAFAC fitting trilinear alternating least squares (TALS) regression procedure, while the second one makes use of the joint approximate diagonalization algorithm. These techniques do not require any knowledge of the propagation channel and/or sensor array manifold and are applicable to a more general class of scenarios than earlier blind approaches to spatial signature estimation.
\end{abstract}

\section{INTRODUCTION}

The use of antenna arrays at base stations has recently gained much interest due to their ability to combat fading, increase system capacity and coverage, and mitigate interference. In the uplink communication mode, signals from different users can be separated at the base station antenna array based on the knowledge of their spatial signatures [1][3]. However, user spatial signatures are usually unknown at the base station and, therefore, have to be estimated.

Traditional (non-blind) approaches to spatial signature estimation make use of training sequences which are periodically transmitted by each user and are known at the base station [2]. However, the use of training sequences reduces the rate of transmission of information symbols and, as a result, blind spatial signature estimation algorithms are of great interest.

There are several blind approaches to spatial signature estimation. The most common one is based on the parametric modeling of spatial signatures using direction of arrival (DOA) parameters [1], [3], [4]. However, these approaches may be highly inefficient because they may involve a high number of parameters and additionally require precise array calibration.

Other popular approaches to blind spatial signature estimation do not use any DOA-based model of spatial signatures but, instead of it, make use of the cyclostationary nature of the communication signals [5] or higher-order statistics [6]. However, the cyclostationarity and non-Gaussianity assimptions may severely limit the practical application of these methods. For example, the method of [5] requires that all users have different cyclic frequencies. The latter condition implies that the users must have different carrier frequencies and/or baud rates [5].

In this paper, we develop a new approach to blind spatial separation using PARAFAC analysis [7]-[10]. A time-varying user power loading is exploited to obtain multiple spatial covariance matrices, as required for the PARAFAC model. Out method is free of any of the aforementioned limitations of the earlier blind techniques.

\section{DATA MODEL}

Let an array of $K$ sensors receive signals from $M$ narrowband sources. The $K \times 1$ snapshot vector of antenna array outputs can be written as

$$
\boldsymbol{y}(n)=\boldsymbol{A} \boldsymbol{s}(n)+\boldsymbol{v}(n)
$$

where $\boldsymbol{A}=\left[\boldsymbol{a}_{1}, \ldots, \boldsymbol{a}_{M}\right] \in \mathbb{C}^{K \times M}$ is the matrix of the user spatial signatures, $\boldsymbol{a}_{m}=\left[a_{1, m}, \ldots, a_{K, m}\right]^{T} \in$ $\mathbb{C}^{K \times 1}$ is the spatial signature of the $m$ th user, $\boldsymbol{s}(n)=$ $\left[s_{1}(n), \ldots, s_{M}(n)\right]^{T} \in \mathbb{C}^{M \times 1}$ is the vector of the user waveforms, $\boldsymbol{v}(n)=\left[v_{1}(n), \ldots, v_{K}(n)\right]^{T} \in \mathbb{C}^{K \times 1}$ is the vector of additive spatially and temporally white Gaussian noise, and $(\cdot)^{T}$ denotes the transpose. Assuming that there is a block of $N$ snapshots available, the model (1) can be written as

$$
\boldsymbol{Y}=\boldsymbol{A} \boldsymbol{S}+\boldsymbol{V}
$$

where $\boldsymbol{Y}=[\boldsymbol{y}(1), \ldots, \boldsymbol{y}(N)] \in \mathbb{C}^{K \times N}$ is the array data matrix, $\boldsymbol{S}=[\boldsymbol{s}(1), \ldots, \boldsymbol{s}(N)] \in \mathbb{C}^{M \times N}$ is the user waveform matrix, and $\boldsymbol{V}=[\boldsymbol{v}(1), \ldots, \boldsymbol{v}(N)] \in \mathbb{C}^{K \times N}$ is the sensor noise matrix. A quasi-static channel is assumed throughout the paper. This assumption means that the spatial signatures are block time-invariant (i.e., the elements of $\boldsymbol{A}$ remain constant over any block of $N$ snapshots).

Assuming that the user signals are uncorrelated with each other and the sensor noise, the array covariance matrix of the received signals can be written as

$$
\boldsymbol{R} \triangleq \mathrm{E}\left\{\boldsymbol{y}(n) \boldsymbol{y}^{H}(n)\right\}=\boldsymbol{A} \boldsymbol{Q} \boldsymbol{A}^{H}+\sigma^{2} \boldsymbol{I}
$$


where $\boldsymbol{Q} \triangleq \mathrm{E}\left\{\boldsymbol{s}(n) \boldsymbol{s}^{H}(n)\right\}$ is the diagonal covariance matrix of the signal waveforms, $\sigma^{2}$ is the sensor noise variance, $\boldsymbol{I}$ is the identity matrix, and $(\cdot)^{H}$ denotes the Hermitian transpose.

The problem studied in this paper is the estimation of the matrix $\boldsymbol{A}$ from noisy array observations $\boldsymbol{Y}$.

\section{PARAFAC MODEL}

First of all, we need to clarify that by identifiability we mean the uniqueness (up to inherently unresolvable source permutation and scale ambiguities) of all user spatial signatures given the exact covariance data. Identifiability in this sense is impossible to achieve with only one known covariance matrix (3) because the matrix $\boldsymbol{A}$ can be estimated from $\boldsymbol{R}_{\mathrm{y}}$ only up to an arbitrary unknown unitary matrix [9]. The approach we will use to provide a unique user spatial signature estimation will be based on an artificial user power loading and PARAFAC analysis. Therefore, next we explain how this model is related to our problem.

Let us divide uniformly the whole data block of $N$ snapshots into $P$ sub-blocks, so that each sub-block contains $N_{s}=\left\lfloor\frac{N}{P}\right\rfloor$ snapshots, where $\lfloor x\rfloor$ denotes the largest integer less than $x$. We fix the transmit power of each user within each sub-block while changing it artificially between different sub-blocks.

We stress that the proposed artificial user power loading does not require precise synchronization among the users, but the users should roughly know the boundaries of epochs over which the powers are kept constant. Therefore, a certain level of user coordination is required. Using the proposed power loading, the received snapshots within any $p$ th block correspond to the following covariance matrix

$$
\boldsymbol{R}(p)=\boldsymbol{A} \boldsymbol{Q}(p) \boldsymbol{A}^{H}+\sigma^{2} \boldsymbol{I}
$$

where $\boldsymbol{Q}(p)$ is the covariance matrix of the user waveforms in $p$ th sub-block. Using all $P$ sub-blocks, we will have $P$ different covariance matrices $\{\boldsymbol{R}(1), \ldots, \boldsymbol{R}(P)\}$. Note that these matrices differ from each other only because the signal waveform covariance matrices $\boldsymbol{Q}(p)$ differ from one sub-block to another.

In practice, the noise power can be estimated and then subtracted from the covariance matrix (4). Let us stack the $P$ matrices $\boldsymbol{R}(p)-\sigma^{2} \boldsymbol{I}, p=1, \ldots, P$ together to form a threeway array $\underline{\boldsymbol{R}}$ which is natural to call the covariance array. The $(i, l, p)$ th element of such an array can be written as

$$
r_{i, l, p} \triangleq\left[\underline{\boldsymbol{R}}_{i, l, p}=\sum_{m=1}^{M} a_{i, m} \nu_{m}(p) a_{l, m}^{*}\right.
$$

where $\nu_{m}(p) \triangleq[\boldsymbol{Q}(p)]_{m, m}$ is the power of the $m$ th user in the $p$ th sub-block and $(\cdot)^{*}$ denotes the complex conjugate. Defining the matrix $\boldsymbol{P} \in \mathbb{R}^{P \times M}$ as

$$
\boldsymbol{P} \triangleq\left[\begin{array}{ccc}
\nu_{1}(1) & \ldots & \nu_{M}(1) \\
\vdots & \ddots & \vdots \\
\nu_{1}(P) & \ldots & \nu_{M}(P)
\end{array}\right]
$$

we can write the following relationship between $\boldsymbol{Q}(p)$ and $\boldsymbol{P}$

$$
\boldsymbol{Q}(p)=\mathcal{D}_{p}\{\boldsymbol{P}\}
$$

for all $p=1, \ldots, P$. In (6), $\mathcal{D}_{p}\{\cdot\}$ is the operator which builds a diagonal matrix by selecting the $p$ th row and putting it on the main diagonal while putting zeros elsewhere.

Equation (5) implies that $r_{i, l, p}$ is a sum of rank-1 triple products. If $M$ is sufficiently small, equation (5) represents a low-rank decomposition of $\underline{\boldsymbol{R}}$. Therefore, the problem of spatial signature estimation can be reformulated as the problem of low-rank decomposition of the three-way covariance array $\underline{R}$.

\section{PARAFAC MODEL IDENTIFIABILITY}

In this section, we study identifiability of the PARAFAC model-based spatial signature estimation. Towards this end, we discuss conditions under which the trilinear decomposition of $\boldsymbol{R}_{\mathrm{y}}$ is unique. Identifiability conditions on the number of sub-blocks and the number of array sensors are derived.

We start with the definition of the matrix Kruskal rank [7].

Definition: The Kruskal rank (or $k$-rank) of a matrix $C$ is $k_{C}$ if and only if every $k_{C}$ columns of $C$ are linearly independent, and either $\boldsymbol{C}$ has $k_{\boldsymbol{C}}$ columns or $\boldsymbol{C}$ contains a set of $k_{C}+1$ linearly dependent columns. Note that $k$-rank is always less than or equal to the conventional matrix rank. It can be easily checked that if $C$ is full column rank, then it is also full $k$-rank.

Using (6) and assuming that the noise term is subtracted from the matrix $\boldsymbol{R}(p)$, we can rewrite (4) as

$$
\boldsymbol{R}(p)=\boldsymbol{A} \mathcal{D}_{p}(\boldsymbol{P}) \boldsymbol{A}^{H}
$$

for all $p=1, \ldots, P$. Let us introduce the matrix

$$
\boldsymbol{R}_{a} \triangleq\left[\begin{array}{c}
\boldsymbol{A D}_{1}(\boldsymbol{P}) \boldsymbol{A}^{H} \\
\vdots \\
\boldsymbol{A D}_{P}(\boldsymbol{P}) \boldsymbol{A}^{H}
\end{array}\right]=(\boldsymbol{P} \odot \boldsymbol{A}) \boldsymbol{A}^{H}
$$

where $\odot$ is the Khatri-Rao (column-wise Kronecker) matrix product [8].

To establish identifiability, we have to obtain under which conditions the decomposition (8) of the matrix $\boldsymbol{R}_{a}$ via matrices $\boldsymbol{P}$ and $\boldsymbol{A}$ is unique (up to scaling and permutation ambiguities). In [7], the uniqueness of trilinear decomposition for the case of real-valued arrays has been established. These results have been later extended to the complex-valued matrix case [10]. In application to our particular PARAFAC problem, the following theorem can be used [13]:

Theorem 1: Consider a set of matrices (7). If for $M>1$

$$
k_{\boldsymbol{A}}+k_{\boldsymbol{P}}+k_{\boldsymbol{A}^{*}}=2 k_{\boldsymbol{A}}+k_{\boldsymbol{P}} \geq 2 M+2
$$

then $\boldsymbol{A}$ and $\boldsymbol{P}$ are unique up to inherently unresolvable permutation and scaling of columns, i.e., if there exists any other pair $\{\overline{\boldsymbol{A}}, \overline{\boldsymbol{P}}\}$ which satisfies (9), then this pair is related to the pair $\{\boldsymbol{A}, \boldsymbol{P}\}$ via

$$
\overline{\boldsymbol{A}}=\boldsymbol{A} \Pi \boldsymbol{\Delta}_{1}, \quad \overline{\boldsymbol{P}}=\boldsymbol{P} \Pi \boldsymbol{\Delta}_{2}
$$

where $\boldsymbol{\Pi}$ is a permutation matrix, and $\boldsymbol{\Delta}_{1}$ and $\boldsymbol{\Delta}_{2}$ are diagonal scaling matrices satisfying

$$
\Delta_{1} \Delta_{1}^{*} \Delta_{2}=\boldsymbol{I}
$$


For $M=1, \boldsymbol{A}$ and $\boldsymbol{P}$ are always unique, irrespectively of (9).

Note that the scaling ambiguity can be easily avoided by taking one of the array sensors as a reference and normalizing user spatial signatures with respect to it. The permutation ambiguity is unremovable but it is usually immaterial because typically the ordering of the estimated spatial signatures is unimportant.

It is worth noting that condition (9) is sufficient for identifiability, and is necessary only if $M=2$ or $M=3$, but is not necessary if $M \geq 4$ [12]. Furthermore, for $M>1$ the condition $k_{\boldsymbol{P}} \geq 2$ becomes necessary [11]. In terms of the number of sub-blocks, the latter condition requires that $P \geq 2$.

The practical conclusion is that in the multiuser case, not less than two covariance matrices must be collected to uniquely identify $\boldsymbol{A}$ which means that the users have to change their powers at least once during the transmission. Similarly, it is necessary that $K>1$.

The following result gives sufficient conditions for the number of sensors to guarantee identifiability.

THEOREM 2: Suppose that

- The elements of $\boldsymbol{A}$ are drawn from distribution $P_{\mathcal{L}}\left(\mathbb{C}^{K M}\right)$, which is assumed continuous with respect to the Lebesgue measure in $\mathbb{C}^{K M}$;

- The elements of $\boldsymbol{P}$ are drawn from distribution $P_{\mathcal{L}}\left(\mathbb{R}^{P M}\right)$, which is assumed continuous with respect to the Lebesgue measure in $\mathbb{R}^{P M}$.

Then

- For $1<M \leq P$, the value of

$$
K \geq \frac{M+2}{2}
$$

is sufficient for almost-sure identifiability [14].

- For $M>P$ and $P \geq 2$, the value of

$$
K \geq \frac{2 M+2-P}{2}
$$

is sufficient for almost-sure identifiability.

PROOF: The assumptions of Theorem 2 mean that the following equalities hold almost surely [14]

$$
\begin{aligned}
& k_{\boldsymbol{A}}=\operatorname{rank}\{\boldsymbol{A}\}=\min (K, M) \\
& k_{\boldsymbol{P}}=\operatorname{rank}\{\boldsymbol{P}\}=\min (P, M)
\end{aligned}
$$

Substituting (12) and (13) into (9), we have

$$
2 \min (K, M)+\min (P, M) \geq 2 M+2
$$

The following cases should be considered:

1) $K \geq M$. In this case, $k_{\boldsymbol{A}}=M$. Furthermore, as $P \geq 2$, we have that $k_{\boldsymbol{P}} \geq 2$. Therefore, condition (14) is always satisfied.

2) $K<M$; $M \leq P$. In this case, $k_{\boldsymbol{A}}=K, k_{\boldsymbol{P}}=M$ and condition (14) becomes

$$
2 K+M \geq 2 M+2
$$

This inequality is equivalent to (10).
3) $K<M$; $M>P$. In this case, $k_{\boldsymbol{A}}=K, k_{\boldsymbol{P}}=P$ and (14) can be written as

$$
2 K+P \geq 2 M+2
$$

This inequality is equivalent to (11).

The proof is complete.

\section{ESTIMATORS}

We will now develop two techniques for blind spatial signature estimation based on the PARAFAC model.

In practice, the exact covariance matrices $\boldsymbol{R}(p)$ are unavailable but can be estimated from the array snapshots $\boldsymbol{y}(n)$, $n=1, \ldots, N$. The sample covariance matrices are given by

$$
\hat{\boldsymbol{R}}(p)=\frac{1}{N_{\mathrm{s}}} \sum_{n=(p-1) N_{\mathrm{s}}+1}^{p N_{\mathrm{s}}} \boldsymbol{y}(n) \boldsymbol{y}^{H}(n), \quad p=1, \ldots, P
$$

These matrices can be used to form a sample three-way covariance array denoted as $\underline{\hat{\boldsymbol{R}}}$.

If $K>M$, then the noise power $\sigma^{2}$ can be estimated as the average of the smallest $K-M$ eigenvalues of the matrix

$$
\tilde{\boldsymbol{R}}=\frac{1}{P} \sum_{p=1}^{P} \hat{\boldsymbol{R}}(p)=\frac{1}{N} \sum_{n=1}^{N} \boldsymbol{y}(n) \boldsymbol{y}^{H}(n)
$$

Then, the estimated noise component $\hat{\sigma}^{2} \boldsymbol{I}$ can be subtracted from sub-blocks of the sample covariance array $\underline{\hat{\boldsymbol{R}}}$.

To formulate our techniques, we will need "slices" of the matrices $\underline{\boldsymbol{R}}$ and $\underline{\hat{\boldsymbol{R}}}$ along different dimensions [10]. That is, let us define the matrices

$$
\boldsymbol{R}_{a}^{(i)} \triangleq\left[r_{i,:,:}\right], \quad \boldsymbol{R}_{b}^{(l)} \triangleq\left[r_{:, l,:}\right], \quad \boldsymbol{R}_{c}^{(p)} \triangleq\left[r_{:,:, p}\right]
$$

where $i, l=1, \ldots, K ; p=1, \ldots, P$; and $r_{i, l, p} \triangleq[\underline{\boldsymbol{R}}]_{i, l, p}$. Similarly,

$$
\hat{\boldsymbol{R}}_{a}^{(i)} \triangleq\left[\hat{r}_{i,:,:]}, \quad \hat{\boldsymbol{R}}_{b}^{(l)} \triangleq\left[\hat{r}_{:, l,:}\right], \quad \hat{\boldsymbol{R}}_{c}^{(p)} \triangleq\left[\hat{r}_{:,:, p}\right]\right.
$$

where $i, l=1, \ldots, K ; p=1, \ldots, P$; and $\hat{r}_{i, l, p} \triangleq[\underline{\hat{\boldsymbol{R}}}]_{i, l, p}$.

For the sake of notation convenience, let us introduce $\boldsymbol{B} \triangleq$ $\boldsymbol{A}^{H}$ and rewrite (8) as

$$
\boldsymbol{R}_{a}=\left[\begin{array}{c}
\boldsymbol{R}_{a}^{(1)} \\
\vdots \\
\boldsymbol{R}_{a}^{(K)}
\end{array}\right]=(\boldsymbol{P} \odot \boldsymbol{A}) \boldsymbol{B}
$$

In the same way, let us define the matrices

$$
\begin{aligned}
& \boldsymbol{R}_{b} \triangleq\left[\begin{array}{c}
\boldsymbol{R}_{b}^{(1)} \\
\vdots \\
\boldsymbol{R}_{b}^{(K)}
\end{array}\right]=\left(\boldsymbol{B}^{T} \odot \boldsymbol{P}\right) \boldsymbol{A}^{T} \\
& \boldsymbol{R}_{c} \triangleq\left[\begin{array}{c}
\boldsymbol{R}_{c}^{(1)} \\
\vdots \\
\boldsymbol{R}_{c}^{(P)}
\end{array}\right]=\left(\boldsymbol{A} \odot \boldsymbol{B}^{T}\right) \boldsymbol{P}^{T}
\end{aligned}
$$


and their sample estimates

$\hat{\boldsymbol{R}}_{a} \triangleq\left[\begin{array}{c}\hat{\boldsymbol{R}}_{a}^{(1)} \\ \vdots \\ \hat{\boldsymbol{R}}_{a}^{(K)}\end{array}\right], \hat{\boldsymbol{R}}_{b} \triangleq\left[\begin{array}{c}\hat{\boldsymbol{R}}_{b}^{(1)} \\ \vdots \\ \hat{\boldsymbol{R}}_{b}^{(K)}\end{array}\right], \quad \hat{\boldsymbol{R}}_{c} \triangleq\left[\begin{array}{c}\hat{\boldsymbol{R}}_{c}^{(1)} \\ \vdots \\ \hat{\boldsymbol{R}}_{c}^{(P)}\end{array}\right]$

Note that for the sake of simplicity, we will not exploit the fact that our PARAFAC model is symmetric. That is, the matrices $\boldsymbol{A}$ and $\boldsymbol{B}$ are assumed to be independent.

\section{A. TALS estimator}

The basic idea behind the TALS procedure for PARAFAC fitting is to update each time a subset of parameters using least square regression while keeping the previously obtained estimates for the rest of parameters fixed. This alternating projection-type procedure is iterated for all subsets of parameters until convergence is achieved [8], [10].

In application to our problem, the PARAFAC TALS procedure can be formulated as follows.

- Step 1: Initialize $\boldsymbol{P}$ and $\boldsymbol{A}$.

- Step 2: Find the estimate of $\boldsymbol{B}$ by solving the following LS problem

$$
\hat{\boldsymbol{B}}=\arg \min _{\boldsymbol{B}}\left\|\hat{\boldsymbol{R}}_{a}-(\boldsymbol{P} \odot \boldsymbol{A}) \boldsymbol{B}\right\|_{F}^{2}
$$

whose analytic solution is given by

$$
\hat{\boldsymbol{B}}=(\boldsymbol{P} \odot \boldsymbol{A})^{\dagger} \hat{\boldsymbol{R}}_{a}
$$

where $(\cdot)^{\dagger}$ denotes the matrix pseudoinverse. Set $\boldsymbol{B}=\hat{\boldsymbol{B}}$.

- Step 3: Find the estimate of $\boldsymbol{A}$ by solving the following LS problem

$$
\hat{\boldsymbol{A}}=\arg \min _{\boldsymbol{A}}\left\|\hat{\boldsymbol{R}}_{b}-\left(\boldsymbol{B}^{T} \odot \boldsymbol{P}\right) \boldsymbol{A}^{T}\right\|_{F}^{2}
$$

whose analytic solution is given by

$$
\hat{\boldsymbol{A}}=\hat{\boldsymbol{R}}_{b}^{T}\left(\left(\boldsymbol{B}^{T} \odot \boldsymbol{P}\right)^{\dagger}\right)^{T}
$$

Set $\boldsymbol{A}=\hat{\boldsymbol{A}}$.

- Step 4: Find the estimate of $\boldsymbol{P}$ by solving the following LS problem

$$
\hat{\boldsymbol{P}}=\arg \min _{\boldsymbol{P}}\left\|\hat{\boldsymbol{R}}_{c}-\left(\boldsymbol{A} \odot \boldsymbol{B}^{T}\right) \boldsymbol{P}^{T}\right\|_{F}^{2}
$$

whose analytic solution is given by

$$
\hat{\boldsymbol{P}}=\hat{\boldsymbol{R}}_{c}^{T}\left(\left(\boldsymbol{A} \odot \boldsymbol{B}^{T}\right)^{\dagger}\right)^{T}
$$

Set $\boldsymbol{P}=\hat{\boldsymbol{P}}$.

- Step 5: Repeat steps 2, 3, and 4 several times (until convergence is achieved) and then compute the final estimate of $\boldsymbol{A}$ as $\hat{\boldsymbol{A}}=\left(\boldsymbol{A}+\boldsymbol{B}^{H}\right) / 2$.

\section{B. Joint Diagonalization-Based Estimator}

Using ideas from [15], we can obtain the estimate of $\boldsymbol{A}$ by means of a joint diagonalizer of the matrices $\boldsymbol{R}(p), p=$ $1, \ldots, P$.

The estimator can be formulated as the following sequence of steps:

- Step 1: Calculate the eigendecomposition of $\tilde{\boldsymbol{R}}$ and find the estimate $\hat{\sigma}^{2}$ of the noise power as the average of the $K-M$ smallest eigenvalues of this matrix.

- Step 2: Compute the whitening matrix as

$$
\boldsymbol{W}=\left[\left(\lambda_{1}-\hat{\sigma}^{2}\right)^{-1 / 2} \boldsymbol{g}_{1}, \ldots,\left(\lambda_{M}-\hat{\sigma}^{2}\right)^{-1 / 2} \boldsymbol{g}_{M}\right]^{H}
$$

where $\left\{\lambda_{m}\right\}_{m=1}^{M}$ are the largest (signal-subspace) eigenvalues of $\tilde{\boldsymbol{R}}$ and $\left\{\boldsymbol{g}_{m}\right\}_{m=1}^{M}$ are the corresponding eigenvectors.

- Step 3: Compute the prewhitened sample covariance matrices as

$$
\hat{\boldsymbol{C}}(p)=\boldsymbol{W} \hat{\boldsymbol{R}}(p) \boldsymbol{W}^{H}, \quad p=1, \ldots, P
$$

- Step 4: Obtain a unitary matrix $\boldsymbol{U}$ as a joint diagonalizer of the set of matrices $\{\hat{\boldsymbol{C}}(p)\}_{p=1}^{P}$.

- Step 5: Estimate the matrix $\boldsymbol{A}$ as

$$
\hat{\boldsymbol{A}}=\boldsymbol{W}^{\dagger} \boldsymbol{U}
$$

Several efficient joint diagonalization algorithms can be used in step 4, (for example, see [16]). However, it should be pointed out that the joint diagonalization-based estimator requires stronger conditions in terms of the number of sensors as compared to the TALS estimator. Indeed, $K \geq M$ is required in the latter technique [15], [16].

\section{Vi. Simulation Results}

In this section, the performance of the developed blind spatial signature estimators is compared with that of the ESPRIT-like estimator [3] and the deterministic Cramer-Rao bound (CRB) of the underlying problem [17].

Although the performance of the proposed blind estimators is independent of array geometry, the ESPRIT-like estimator is based on the uniform linear array (ULA) assumption. Therefore, to compare the estimators in a proper way, we assume a ULA of $K=10$ omnidirectional sensors spaced half a wavelength apart, and $M=2$ BPSK user signals impinging on the array from $\theta_{1}=-50^{\circ}$ and $\theta_{2}=15^{\circ}$ relative to the broadside. $P=10$ sub-blocks are used in our techniques. The user powers are changed between different sub-blocks uniformly with the power change factor (PCF) of 1.2 , where $\boldsymbol{P}=\operatorname{SNR}(\boldsymbol{E}+\mathrm{PCF} \cdot \boldsymbol{D})$ and it is assumed that $\sigma^{2}=1$. Here, SNR is the average user SNR in a single sensor, $\boldsymbol{E}$ is the matrix whose elements are all equal to one, and $D$ is a random matrix whose elements are uniformly and independently drawn from the interval $[-0.5,0.5]$.

The estimator performance is compared in terms of the root mean square error (RMSE)

$$
\mathrm{RMSE}=\sqrt{\frac{1}{L M K} \sum_{l=1}^{L}\|\hat{\boldsymbol{A}}(l)-\boldsymbol{A}\|_{F}^{2}}
$$




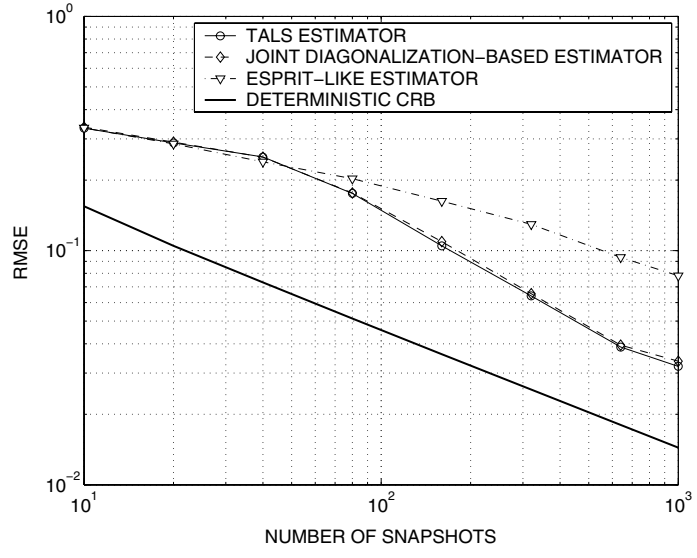

Fig. 1. RMSEs versus $N$.

where $L=100$ is the number of independent simulation runs and $\hat{\boldsymbol{A}}(l)$ is an estimate of $\boldsymbol{A}$ obtained from the $l$ th run. Note that the scaling and permutation ambiguities have been eliminated by means of a proper ordering and normalizing the columns of $\hat{\boldsymbol{A}}(l)$.

Following [3], we assume that the array gains and phases are unknown, i.e., the received data are modeled as (2) with

$$
\boldsymbol{A}=\boldsymbol{\Gamma} \boldsymbol{A}_{0}
$$

where $\boldsymbol{A}_{0}$ is the matrix of nominal plane-wavefront user spatial signatures and $\boldsymbol{\Gamma}$ is the diagonal matrix containing the array unknown gains and phases, i.e., $\Gamma(\gamma)=$ $\operatorname{diag}\left\{g_{1} e^{j \phi_{1}}, \ldots, g_{K} e^{j \phi_{K}}\right\}$. The unknown gains $g_{1}, \ldots, g_{K}$ are independently drawn in each simulation run from the uniform random generator with the standard deviation equal to one, while the unknown phases $\phi_{1}, \ldots, \phi_{K}$ are independently and uniformly drawn from the interval $[0,2 \pi)$.

Figure 1 displays the RMSEs of the estimators tested and the $\mathrm{CRB}$ versus $N$ for $\mathrm{SNR}=10 \mathrm{~dB}$. Figure 2 shows the performances of the same estimators and the $\mathrm{CRB}$ versus the SNR for $N_{s}=100$.

We observe that both PARAFAC model-based estimators perform substantially better than the ESPRIT-like estimator. However, none of the estimators tested achieves the CRB.

\section{CONCLUSIONS}

The problem of blind user spatial signature estimation using the parallel factor (PARAFAC) analysis model has been addressed. A time-varying user power loading in the uplink mode has been proposed to make the problem identifiable and to enable the application of the PARAFAC analysis model. Identifiability issues have been studied and two blind spatial signature estimation algorithms have been developed. These techniques have better performance than other known blind approaches and are applicable to a more general class of scenarios.

\section{REFERENCES}

[1] D. Astèly, B. Ottersten and A. L. Swindlehurst, "Generalised array manifold model for wireless communication channel with local scattering," IEE Proc. Radar, Sonar Navigation, vol. 145, pp. 51-57, Feb. 1998.

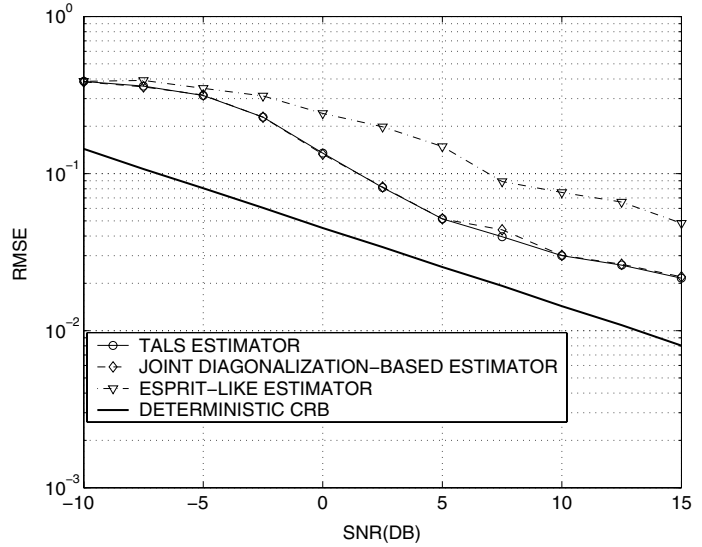

Fig. 2. RMSEs versus SNR.

[2] A. L. Swindlehurst, "Time delay and spatial signature estimation using known asynchronous signals," IEEE Trans. Signal Processing, vol. 46, pp. 449-462, Feb. 1998

[3] D. Astèly, A. L. Swindlehurst and B. Ottersten, "Spatial signature estimation for uniform linear arrays with unknown receiver gains and phases," IEEE Trans. Signal Processing, vol. 47, pp. 2128-2138, Aug. 1999.

[4] A.J. Weiss and B. Friedlander, “'Almost blind' steering vector estimation using second-order moments," IEEE Trans. Signal Processing, vol. 44, pp. 1024-1027, Apr. 1996.

[5] Q. Wu and K. M. Wong, "Blind adaptive beamforming for cyclostationary signals," IEEE Trans. Signal Processing, vol. 44, pp. 2757-2767, Nov. 1996.

[6] J.-F. Cardoso and A. Souloumiac, "Blind beamforming for non-Gaussian signals," IEE Proc., vol. F-140, no. 6, pp. 362-370, Dec. 1993.

[7] J. B. Kruskal, "Three-way arrays: rank and uniqueness of trilliner decompositions, with application to arithmetic complexity and statistics," Linear Algebra Application, vol. 16, pp. 95-138, 1977.

[8] N. D. Sidiropoulos, R. Bro and G. B. Giannakis, "Parallel factor analysis in sensor array processing," IEEE Trans. on Signal Processing, vol. 48, No. 8, pp. 2377-2388, Aug. 2000.

[9] N. D. Sidiropoulos and R. Bro, "On the uniqueness of multilinear decomposition of N-way arrays," J. Chemometrics, vol. 14, pp. 229-239, 2000.

[10] N. D. Sidiropoulos, G. B. Giannakis and R. Bro, "Blind PARAFAC receivers for DS-CDMA systems," IEEE Trans. Signal Processing, vol. 48, No.3, pp. 810-823, Mar. 2000.

[11] R. L. Harshman, "Determination and proof of minimum uniqueness conditions for PARAFAC1," UCLA Working Papers in Phonetics, vol. 22, pp. 111-117, 1972.

[12] J.M.F. ten Berge, and N.D. Sidiropoulos, "On Uniqueness in CANDECOMP/PARAFAC," Psychometrika, vol. 67, No. 3, Sep. 2002.

[13] J.M.F. ten Berge, N.D. Sidiropoulos, and R. Rocci "Typical rank and INDSCAL dimentionality for symmetric three-way arrays of order $\mathrm{I} \times 2 \times 2$ or I $\times 3 \times 3$," Linear Algebra and its Applications, to appear.

[14] T. Jiang, N. D. Sidiropoulos, and J. M. F. ten Berge "Almost-sure identifiability of multudimentional harmonic retrieval," IEEE Trans. Signal Processing, vol. 49, pp. 1849-1859, Sept. 2001.

[15] A. Belouchrani, K. Abed-Meraim, J-F. Cardoso and E. Moulines, "A blind source separation technique using second-order statistics," IEEE Trans. on Signal Processing, vol. 45, No.2, pp. 434-444, Feb. 1997.

[16] A. Yeredor, "Non-orthogonal joint diagonalization in the least-squares sense with application in blind source separation," IEEE Trans. Signal Processing, vol. 50, pp. 1545-1553, July 2002.

[17] Y. Rong, S. A. Vorobyov, A. B. Gershman, and N. D. Sidiropoulos, "Blind spatial signature estimation via time-varying user power loading and parallel factor analysis," submitted to IEEE Trans. Signal Processing, 2003. 